\title{
Molecular dynamic simulation of complex NS2B-NS3 DENV2 protease with potential inhibitors of cyclic tripeptide
}

\author{
Usman Sumo Friend Tambunan*, Irwan Kurniawan and Arli Aditya Parikesit \\ Department of Chemistry, Faculty of Mathematics and Science, University of Indonesia Depok 16424, Indonesia. \\ Accepted 5 July, 2013
}

\begin{abstract}
The pathogenic dengue virus (DV) is a growing global threat for which there is no specific treatment and prevention. Several vaccines have been developed against the disease, but they only prevent the disease and reduce the risk of death. Consequently, the antiviral drug becomes the most powerful treatment to solve this problem. The virus possesses a two component NS2B-NS3 protease that cleaves viral precursor proteins, and therefore represents a target for the development of antiviral drugs. In many researches, several models of peptides inhibitor were generated in complexes with the NS2B-NS3 DENV2 protease by performing molecular docking. The goal of this research was to study the interaction of ligands as inhibitors for protein (enzyme) in solvent explicit condition by performing molecular dynamics simulation at 300 and $312 \mathrm{~K}$. The simulations were performed on four disulfide cyclic tripeptide ligands (RGR, KRK, RRA and RKR) and two proline-proline based cyclic peptide ligands (RKK and KKR) compared with linear peptide inhibitor Bz-Nle-K-R-R-H as standard ligand. The results provide conformational changes of enzyme-inhibitor complex that is shown by root-meansquare deviation (RMSD) values. These results show that dynamic behavior of the complex occurs in the presence of solvent. The interaction between ligand and amino acid residue of enzyme changed during simulation. By these parameters, RKR becomes the best ligand compared with others. The ligand has hydrogen bond interaction with the residue of active side, and is stable during simulation at both temperatures. In conclusion, RKR ligand is proposed as an antiviral drug by performing molecular dynamics simulation.
\end{abstract}

Key words: Dengue virus, DENV2, NS2B-NS3 protease, inhibitor, cyclic peptide, ligand, molecular dynamics simulation, interaction, conformation, drug.

\section{INTRODUCTION}

Dengue fever is a disease caused by dengue viruses that infect humans and has been affecting a significant majority of the world's population (WHO, 2010). Dengue virus (DENVs) comes from the family Flaviviridae, and genus Flavivirus (Tomlinson et al., 2009). Dengue virus is transmitted by Aedes mosquitoes through their bites.
Female mosquito Aedes aegypti and Aedes albopictus are major vectors of dengue virus. Based on sequence similarities and differences in antigenic properties, this virus has four serotypes, namely: DENV-1, DENV-2, DENV-3 and DENV-4 (Lescar et al., 2008). Infection by one of these serotypes cannot protect against other

${ }^{*}$ Corresponding author. E-mail: usman@ui.ac.id.

Abbreviations: DENVs, Dengue virus; DSS, dengue shock syndrome; NS, nonstructural; RMSD, root mean square deviation. 
serotypes infection, but may increase the pathogenicity of subsequent infection by the three other serotypes (Tomlinson et al., 2009). Intial infection of dengue virus has caused the death rate of about $5 \%$, and increase to $40 \%$ if a virus infection continues to progress until the dengue shock syndrome (DSS) (Tambunan et al., 2009). Currently, available treatment is only to reduce symptoms and the risk of death. Therefore, antiviral medication needs to be developed (Sampath, 2009). Every stage, from the infection of host cells to the assembly of new virus particles, can be targeted in the development of the inhibitor molecule (drug design) that can inhibit the activity of replication enzymes of the dengue virus, such as the enzyme protease, RdRP, methyltransferase and helicase (Kirsten, 2008).

A peptide that has the $\alpha$-keto amide bond in its amides can act as a competitive inhibitor of the NS3 protease with a $\mathrm{K}_{\mathrm{i}}$ value in the micro-molar region (Katzenmeier, 2004). Some examples of nonstructural (NS) enzymes of the replication complex is a cofactor of NS2B-NS3 protease, NS3 helicase / nucleoside triphosfatase (NTPase) / RNA 5 'triphosfatase (RTPase), and NS5 methyltransferase / RNA-dependent RNA polymerase (RdRp). These proteins can be used as potential inhibitor targets for antiviral agents during virus replication (Tomlinson et al., 2009). NS3 protease enzymes are the primary targets for the development of antiviral drugs since dengue NS2B-NS3 protease is required for viral replication (Othman et al., 2007). NS3 protease's active sides are at residues His51, Asp75 and Ser135. NS3 protease enzyme requires NS2B as a cofactor to activate the protease enzyme (Qi et al., 2007). The 3D peptide database content has expanded the role of computational high throughput screening (HTS) in the search for dengue antiviral drug (Tomlinson et al., 2009). There are many computational studies that have been carried out by identifying the corresponding protein targets, and designing an inhibitor that can interact with the target protein in order to block the normal function of the target (Singh, 2006). The studies of genomics and proteomics have facilitated the discovery of new targets for drug design (Alonso et al., 2006). Molecular modeling is a technique for designing and displaying the structure and properties of certain molecules by using computational chemistry and graphical visualization techniques (Leach, 2001). Computational methods in drug design was utlized because it is more rational and efficient (Tomlinson et al., 2009). Molecular modeling techniques include molecular docking and molecular dynamics. According to Nurbaiti (2009), in his research, biomolecules such as proteins and nucleic acids is a dynamic system.

Research conducted by Yin (2005) aimed to find NS3 protease inhibitors that are potent and selective based on the evaluation of the synthesis of tetra peptide sequence of linear aldehyde. Synthesis of the evaluation results showed that the shape inhibitor Bz-Lys-Arg-NLE-Arg-H $\left(\mathrm{K}_{\mathrm{i}} 5.8 \mu \mathrm{M}\right)$ is the best inhibitor and is active in dengue virus NS3 protease. Research carried out by Tambunan et al. $(2009,2010)$ on NS3-NS2B protease inhibitors showed that cylic pentapeptide are indeed feasible for inhibitors. Thus, it is possible to study the effect of solvent molecules in protein structures (Alonso et al., 2006). However, the decision to pick tripeptide as the research object was chosen due to the previous study of our group (Jois et al., 1996)

The purpose of this research was to study the interactions and conformations of the seven tripeptide potential inhibitors with dengue NS2B-NS3 protease virus type 2 in the hydrated state using the method of molecular dynamics simulations. In addition, this study also evaluates and determines potential ligands to be developed as a drug.

\section{MATERIALS AND METHODS}

Following the study of Usman et al. (2011, 2010), this study's methodology was divided into two parts: the molecular docking and dynamics session.

\section{D Structure of dengue virus NS2B-NS3 protease}

Complex three-dimensional structure of the NS2B-NS3 protease dengue virus was obtained from the database of Protein Data Bank (PDB) with the specific enzyme ID 2FOM (http://www.rcsb.org/pdb/).

\section{The active visualization of NS2B-NS3 protease}

Visualization of the active dengue virus NS3 protease and the location of NS2B as cofactor was done by using MOE2008.10 software.

Geometry optimization and energy minimization of the three dimensional structure of NS2B-NS3 protease

The process of geometry optimization and energy minimization of NS2B-NS3 protease was made by removing water molecules and chlorine ions. Furthermore, protonation with protonate 3D was done to change the state into the ionization level.

Design of three dimensional structure of cyclical peptide inhibitors

At this stage, ACDlabs software was used to design the cyclic peptide amino acid sequences into 3D structure. Cyclic peptides modeled with each end of the cysteine residues were added to form disulfide bridges and proline bond to yield a cyclic peptide based on the proline-proline bridge. Furthermore, the model peptide was conditioned in a state of zwitter ion.

Geometry optimization and energy minimization of the three dimensional structure of cyclical peptide

Cyclic peptide optimization was carried out on the MOE database viewer (dv). The optimization process begins with 'wash' process for 
the entire cyclic peptide. Then, the partial charge was applied with MMFF94 method.

\section{Molecular docking}

Molecular docking process was done using the MOE 2008.10 software through the MOE-dock program. Placement methods used are matcher triangle with the 2500000 rounds. Scoring function used is the London dG, while the refinement was done using force field with the population of 1000 repetitions. The tripeptides were made in the restriction of the active residues.

\section{Ligand interactions at a temperature of 300 and $312 \mathrm{~K}$}

Analysis of ligand interactions with the enzyme at temperatures of $312 \mathrm{~K}$ as well as the analysis of ligand interactions at $300 \mathrm{~K}$ was done by LigX ligand Interaction option in the MOE 2008.10 software.

Enzyme-ligand complex conformation of the molecular dynamic results

In the initialization phase, the observations were taken every $10 \mathrm{ps}$, then the heating stage was conducted every $10 \mathrm{ps,} \mathrm{equilibration}$ stage of $500 \mathrm{ps}$, and cooling stages of $5 \mathrm{ps}$.

\section{RESULTS AND DISCUSSION}

\section{D Structure of dengue virus NS2B-NS3 protease}

The selection of the specific PDB code 2FOM is based on the structure of the NS3 protease interactions with NS2B as a cofactor, though 2FOM is the only PDB code of NS2B-NS3 protease derived from dengue virus type 2 (Erbel et al., 2006).

\section{Visualization of active NS3 protease and NS2B cofactor}

NS3 serine protease has active sides in the three functional amino acid, namely His51, Asp75 and Ser135 (Wichapong et al., 2009). These three amino acids are present in the enzyme binding site and has polar and hydrophilic properties. NS2B-NS3 protease has a relatively flat binding site (Erbel et al., 2006). This active site has a negatively charged region around Asp75 and Ser135. Moreover, these enzymes prefer substrates that have a positive residual group (Noble et al., 2010). Visualization results of three active side residues can be done using MOE 2008.10 software, and its interaction with the cofactor is shown in Figure 1. The far distance of NS2B from the active side showed that the cofactor does not contribute directly to the enzyme catalytic reaction, but plays an important role in enzyme catalytic activity (Lescar et al., 2008). The active form of protease can occur if the cofactor NS2B hydrophilic 49 to 95 amino acid residues interact with the NS3 protease through nine residues connecting flexible Gly -Ser-Gly $_{4}$ (Lescar et al., 2008; Leung et al., 2001). The data were adopted from in vitro studies by Yusof et al. (2000). Research conducted by Erbel et al. (2006) reveals that the productive conformation of the NS2B-NS3 protease in the catalytic process makes this enzyme an important target in drug discovery. NS2B-NS3 protease chymotrypsin fold adopted a conformation of two $\beta$-barrel that surrounds the three active sides. Each $\beta$-barrel is formed by six $\beta$-strands (Bollati et al., 2010). Therefore, these forms can protect hydrophobic residues from the solvent and provide stability to the protease (Lescar et al., 2008; Erbel et al., 2006). In addition, amino acid residues Arg78-Leu87 of NS2B cofactor also influences the active side formation of the $\beta$-hairpin that was inserted into the end of the Cterminal $\beta$-barrel. In this end, more active side residues concentrate on the introduction of the substrate (Lescar et al., 2008).

\section{Geometry optimization and energy minimization of the 3D structure of NS2B-NS3 protease}

The protonation of NS2B-NS3 protease complex is carried out with the program protonate3D of MOE 2008.10 software. This process was done to condition the enzyme in the protonated state (Tambunan et al., 2010). In the next phase, the addition of the enzyme complex loads (partial charge) using the current method of force field parameters was done. The last stage of the process of geometry optimization and minimization of enzymes is energy minimization using the software MOE 2008.10. Energy minimization can eliminate undesirable interactions (bad contact) of a structure (Nurbaiti, 2009). Use of force field MMFF94x is considered better than the other force field due to a better sensitivity to the geometry optimization of protein (enzyme) with the inhibitor (Tambunan et al., 2010). Energy minimization process was done by the RMS gradient $0.05 \mathrm{kcal} / \AA$ corresponding to the protein. Other parameters in the energy minimization process were done using the default standard of MOE2008.10.

\section{Molecular docking}

The selected seven ligands were performed with molecular docking processes; these include the standard ligand Bz-Lys-Arg-NLE-Arg- $\mathrm{H}$, ligand KRK, RGR, KKR, RKK, RRA and RKR. They were performed to dock with the three sides of the enzyme active NS3 protease of His51, Asp75 and Ser135. The scoring functions in MOE 2008.10 software is based on the function of force-field scoring. This function takes into account the ligandreceptor interaction energy and ligand internal energy (Nylander, 2007). In this study, the utlized scoring function is the London $\mathrm{dG}$. This scoring function estimates the Gibbs free energy of binding $\left(\Delta G_{\text {binding }}\right)$ by posing a ligand to an enzyme (Vilar et al., 2008). Triangle matcher 


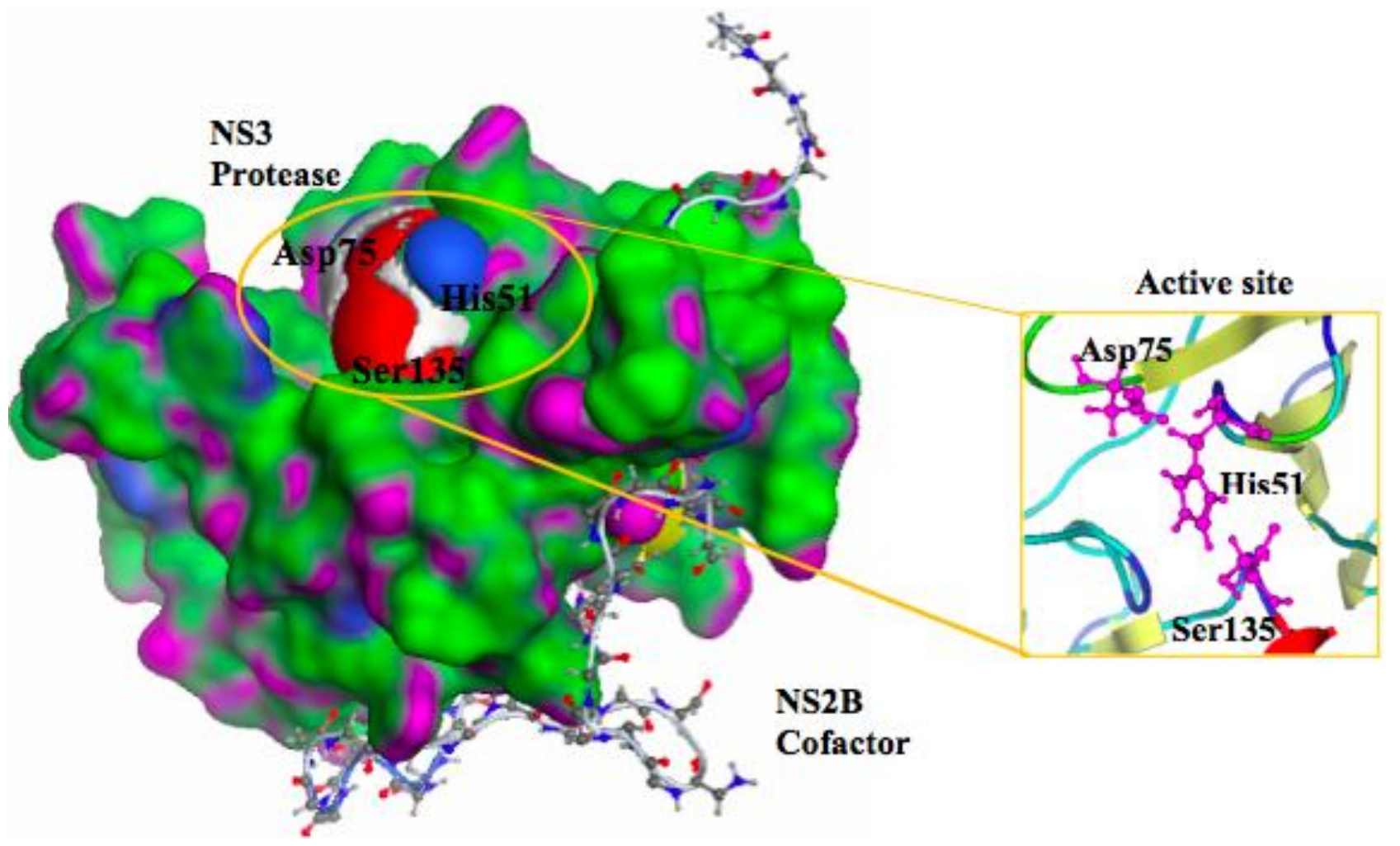

Figure 1. Visualization of the active NS3 protease and NS2B cofactor.

Table 1. The results of molecular docking.

\begin{tabular}{lccc}
\hline Ligand & $\mathbf{\Delta G}_{\text {binding }}$ (kkal/mol) & $\mathbf{p K}_{\mathrm{i}}$ & Log $\mathbf{P}$ \\
\hline Cyclic (-Cys-RKR-Cys-) & -16.493 & 12.231 & -4.110 \\
Cyclic (-Cys-RRA-Cys-) & -15.417 & 11.246 & -3.721 \\
Cyclic (-Cys-KRK-Cys-) & -14.436 & 10.864 & -2.746 \\
Cyclic (-Cys-RGR-Cys-) & -12.549 & 9.977 & -4.110 \\
Cyclic (-Pro-RKK-Pro-) & -12.209 & 9.121 & -2.042 \\
Cyclic (-Pro-KKR-Pro-) & -12.182 & 8.645 & -2.042 \\
Bz-Nle-Lys-Arg-Arg-H & -10.467 & 7.940 & -0.716 \\
\hline
\end{tabular}

is used to orient the ligand in the active side groups based on the charge and spatial fit to produce an optimal bonding orientation. The force field utilized the Generalized Born model of solvation on the stage of final energy evaluation while GridMIn uses electrostatic calculations on the minimization process (Villar et al., 2008).

\section{Analysis of molecular docking}

The molecular docking process data are shown in Table 1. Display of residual interactions and hydrogen bonding contacts between the ligand and the enzyme is shown in
Figure 2. The data of the interaction of residue contacts and hydrogen bonding are shown in Table 2 . In Table 1, it is shown that the $\Delta \mathrm{G}_{\text {binding }}$ values are negative indicating that a complex between the ligand and the enzyme can be formed. RKR ligands in the table have the greatest value of $\Delta G_{\text {binding }}(-16.493 \mathrm{kcal} / \mathrm{mol})$ when compared with the other six ligands. In contrast, the standard ligand produces the smallest value of $\Delta G_{\text {binding }}(-10.467$ $\mathrm{kcal} / \mathrm{mol})$. This indicates that the ligand bound RKR is the most powerful among the other six ligands. Table 2 on the block letters indicate the active residues of the NS3 protease. Overall, the data shows that the molecular docking of ligands formed seven interaction with the enzyme active NS3 protease. As shown in Figure 2, the 
Table 2. Interaction results of molecular docking.

\begin{tabular}{|c|c|c|}
\hline Ligand & Contact residue & Hydrogen bond \\
\hline (-Cys-RKR-Cys-) & $\begin{array}{l}\text { Trp50, Val52, Asp75, Pro132, Gly133, His51, } \\
\text { Lys73, Asn152, Gly153, Val162, Val154, Tyr161, } \\
\text { Leu128 }\end{array}$ & $\begin{array}{l}\text { Val52, Asp75, His51, Lys73, } \\
\text { Asn152, Gly153, Val154, Val162 }\end{array}$ \\
\hline (-Cys-RRA-Cys-) & $\begin{array}{l}\text { Pro132, His51, Gly153, Asn152, Lys73, Asp75, } \\
\text { Val72, Trp50, Arg54 }\end{array}$ & $\begin{array}{l}\text { Pro132, His51, Gly153, Lys73, } \\
\text { Asp75, Val72, Trp50, Arg54 }\end{array}$ \\
\hline (-Cys-KRK-Cys-) & $\begin{array}{l}\text { Arg54, Asp75, Gly153, Asn152, Leu128, Ser135, } \\
\text { Tyr150, Gly151, Pro132, His51, Gly133, Val52, } \\
\text { Ile36 }\end{array}$ & $\begin{array}{l}\text { Arg54, Asp75, Gly153, Asn152, } \\
\text { Tyr150, Pro132, His51, Val52, } \\
\text { lle36 }\end{array}$ \\
\hline (-Cys-RGR-Cys-) & $\begin{array}{l}\text { Gln35, Glu92, Ile36, Pro132, Val52, His51, Arg54, } \\
\text { Asp75, Asn152, Gly153 }\end{array}$ & $\begin{array}{l}\text { Gln35, Val52, His51, Arg54, } \\
\text { Asp75, }\end{array}$ \\
\hline (-Pro-RKK-Pro-) & $\begin{array}{l}\text { Val52, His51, Leu128, Ser163, Gly151, Asn152, } \\
\text { Asp75, Gly153, Lys74, Lys73, Val72 }\end{array}$ & $\begin{array}{l}\text { Val52, His51, Gly151, Asn152, } \\
\text { Gly153, Lys73, Val72 }\end{array}$ \\
\hline (-Pro-KKR-Pro-) & $\begin{array}{l}\text { Val72, Asp75, Asn152, Trp50, Gly153, His51, } \\
\text { Ile36, Pro132, Gly133 }\end{array}$ & $\begin{array}{l}\text { Val72, Asp75, Asn152, Gly153, } \\
\text { His51, Ile36, Pro132, }\end{array}$ \\
\hline Bz-Nle-Lys-Arg-Arg-H & $\begin{array}{l}\text { Arg54, Asp75, Val52, Ile36, His51, Gly133, Gln27, } \\
\text { Ser135, Pro132, Gly153, Asn152 }\end{array}$ & $\begin{array}{l}\text { Asp75, lle36, His51, Gln27, } \\
\text { Pro132, Gly153 }\end{array}$ \\
\hline
\end{tabular}

Bold: Active site residues that facilitate hydrogen bonding with the ligand.

a)

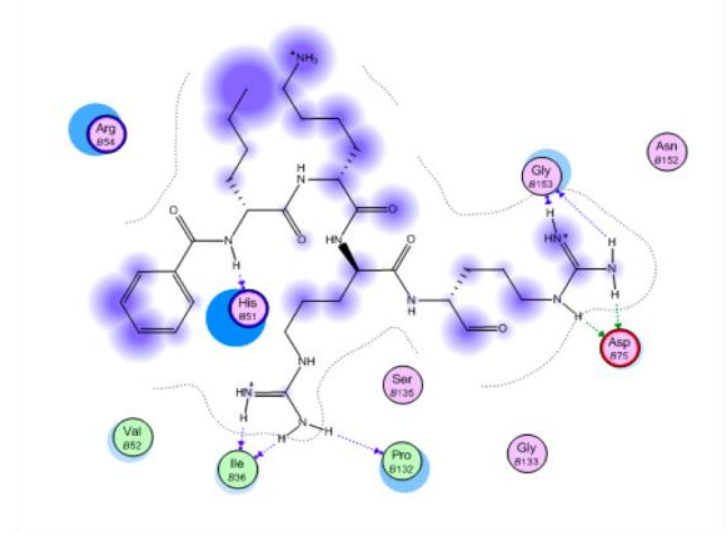

b)

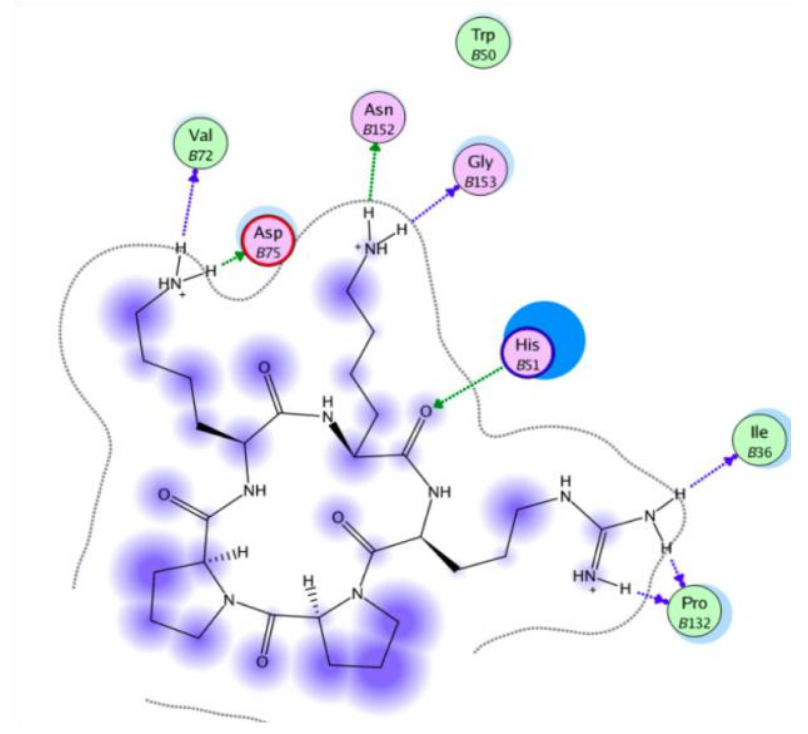

Figure 2. Results of residue contacts ligand docking: (a) Standard ligand, (b) RKK ligand, (c) KKR ligand, (d) ligand RGR; (e) ligand KRK; (f) ligand RRA; (g) ligand RKR.

visualization of RKR data on the ligands can form two hydrogen bonds to the active side residues Asp75 and two hydrogen bonds to the His51 residue, while the ligand RRA only forms a hydrogen bond to the Asp75 residue and two hydrogen bonds to the His51 residue (Tambunan et al, 2010; Nurbaiti, 2009). Table 1 also consist of $\log P$ values for the seven ligands. From Table 1, it is shown that the design of cyclic peptides has a nega- 
c)

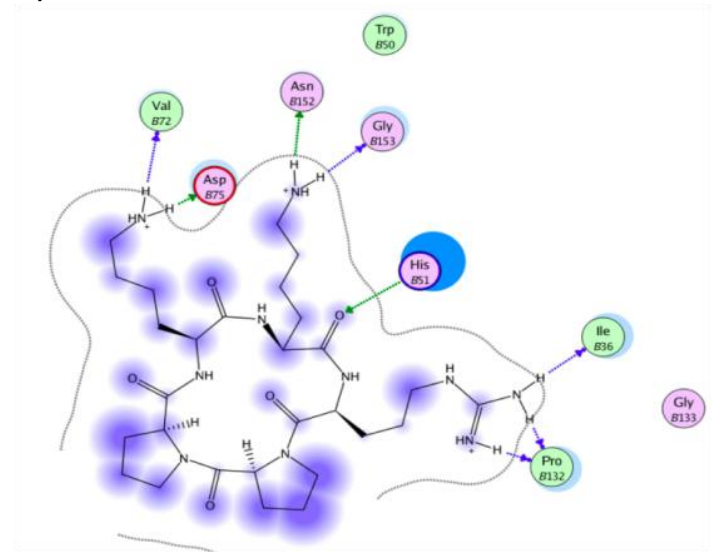

d)

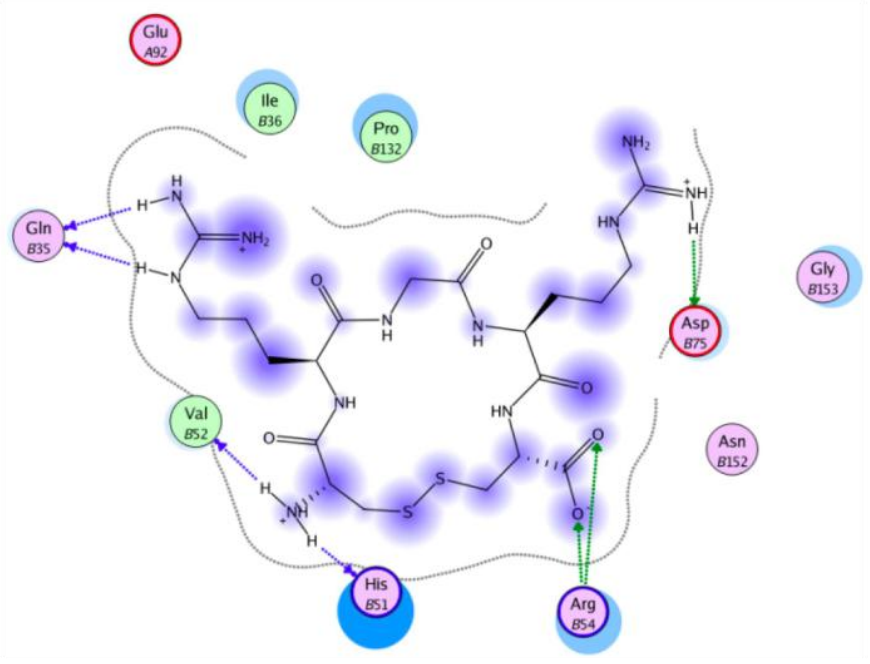

Figure 2. Contd.

e)

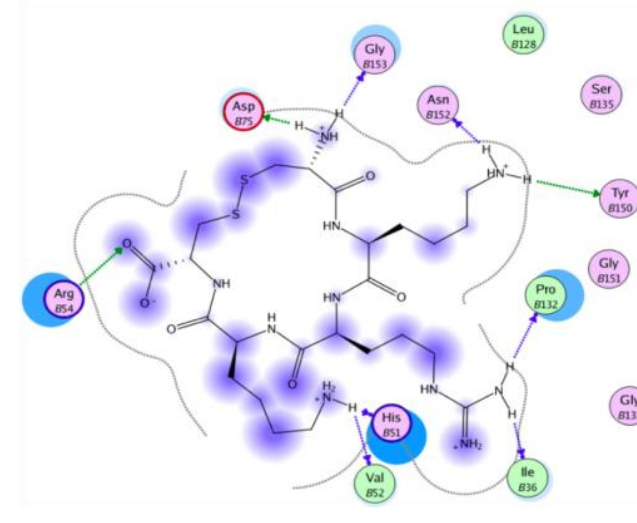

f)

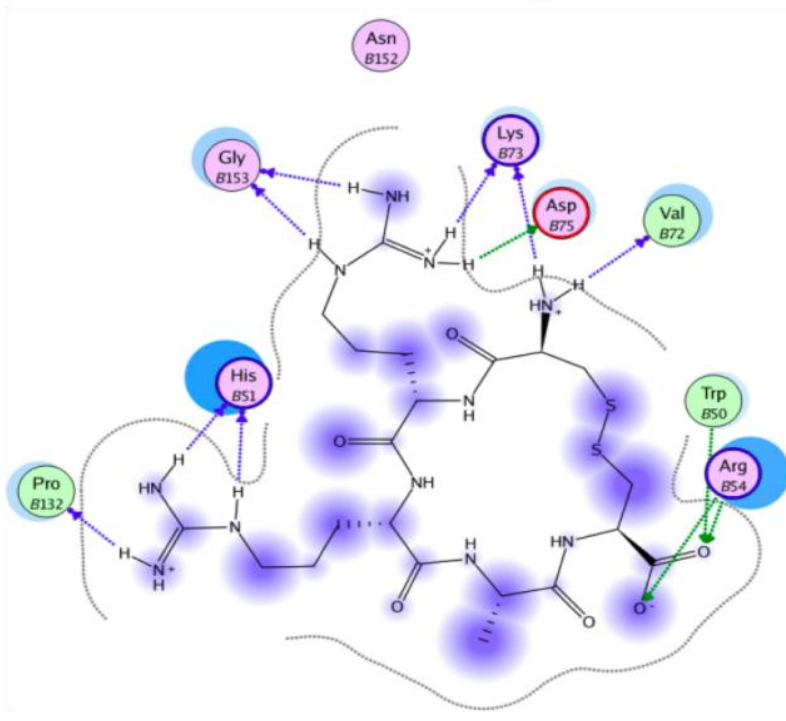

Figure 2. Contd.

tive $\log P$ value. Based on the data in Tables 1 and 2 , the molecular docking of ligand candidates has produced seven RKRs as the best ligands. They are based on the value of $\Delta \mathrm{G}_{\text {binding }}-16.493 \mathrm{kcal} / \mathrm{mol}$ and the $\mathrm{p} \mathrm{Ki}$ of 12.231 . Those values are considered the best when compared with the seven other ligands.

\section{Molecular dynamics simulations}

Molecular dynamics simulations were performed using the software program of dynamic MOE.2008.10. This process uses Nosé-Poincaré-Andersen (NPA) algorithm that can be properly ensembled, and ensure that the trajectory is generated according to the desired thermodynamic conditions. The whole stage setting was done on the potential parameter setup software contained in MOE.2008.10 (Alonso et al., 2006). The force field utilized MMFF94x and NVT ( $\mathrm{N}=$ constant number, $\mathrm{V}=$ volume, and $\mathrm{T}$ =temperature) ensemble, and a pressure of $101 \mathrm{kPa}$. According to Tambunan et al. (2010), force field MMFF94x can put hydrogen atoms on the most appropriate position. In addition, the pocket is also set at a cutoff of $6 \AA$. The system begins with the initiali- 


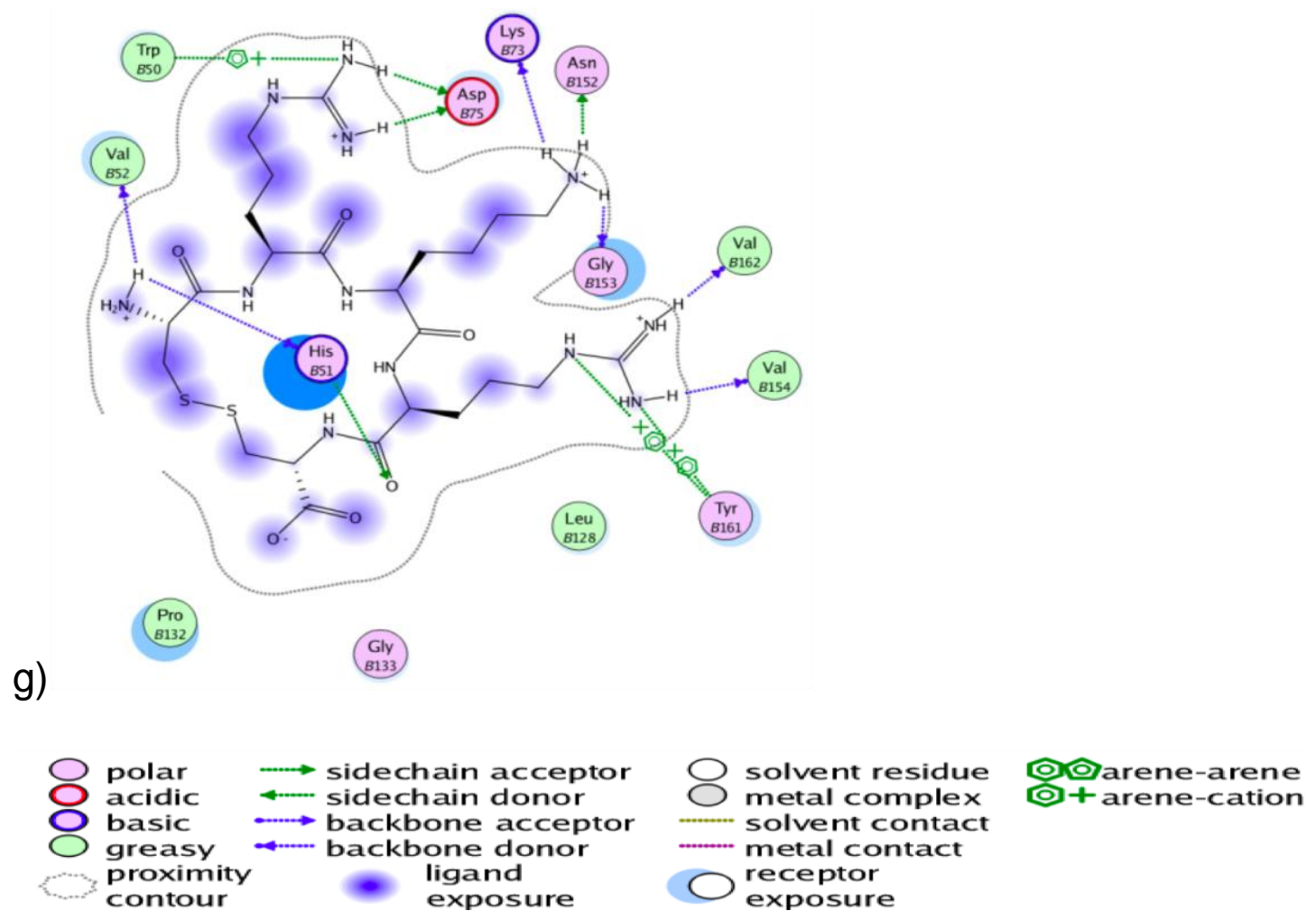

Figure 2. Contd.

zation phase. Initialization molecules include the determination of the position and initial velocity of the molecules. At this stage of initialization, simulations were performed for 50 ps; correlation plot can be seen in Figure 3. From these images, it can be observed that at 50 ps, the potential energy of the system tends to be stable and it was chosen as the initial stage of the primary simulation for 5000 ps. Furthermore, the main stage during the 5000 ps simulations was performed at the temperatures of 300 $\mathrm{K}$ and $312 \mathrm{~K}$.

\section{Molecular dynamics simulations at $300 \mathrm{~K}$ temperature}

Temperature of $300 \mathrm{~K}$ was chosen because it is a room temperature. The time setting was adjusted at dynamics box column with the run process for 5000 ps. The cooling stage performed for 20 ps until the simulation increased to a temperature of $1 \mathrm{~K}$. Then, the tool box for the data storage was selected for position, velocity, and acceleration in every $0.5 \mathrm{ps}$.

\section{Molecular dynamics simulation on the temperature of $312 \mathrm{~K}$}

Temperature of $312 \mathrm{~K}$ was chosen on the grounds that the condition is the same as the temperature of a person who has dengue fever (Tambunan et al., 2009). Heating stage process was done to raise the temperature of the system towards a state of equilibrium stage. The equilibrium state is where the density of distribution of the atomic positions in all the systems that provide relaxed conditions on the molecule is restrained when the system is heated (Tambunan et al, 2010; Nurbaiti, 2009). According to Sharma et al. (2009), obtained trajectory is formed by recording the coordinates of the various structural changes within a certain time scale showing the position of each atom.

\section{Analysis of molecular dynamics simulation}

The molecular dynamics simulation was performed with a $5 \mathrm{~ns}$ time scale. The treatment time of $5 \mathrm{~ns}$ was adopted from studies conducted by Nurbaiti (2009) and Tambunan (2010) which suggest that the molecular dynamics simulation was performed on a nanosecond time scale $\left(10^{-9}\right)$. In the initialization phase, the six frames were observed in every 10 ps simulation. To obtain a heating process, three frames was observed every $10 \mathrm{ps.} \mathrm{On} \mathrm{the}$ main stage of the simulation, the observations were made every 500 ps which produces 11 frames of a conformational change. For the cooling phase, five frames were observed every $5 \mathrm{ps}$. Observations on the conformational 
U

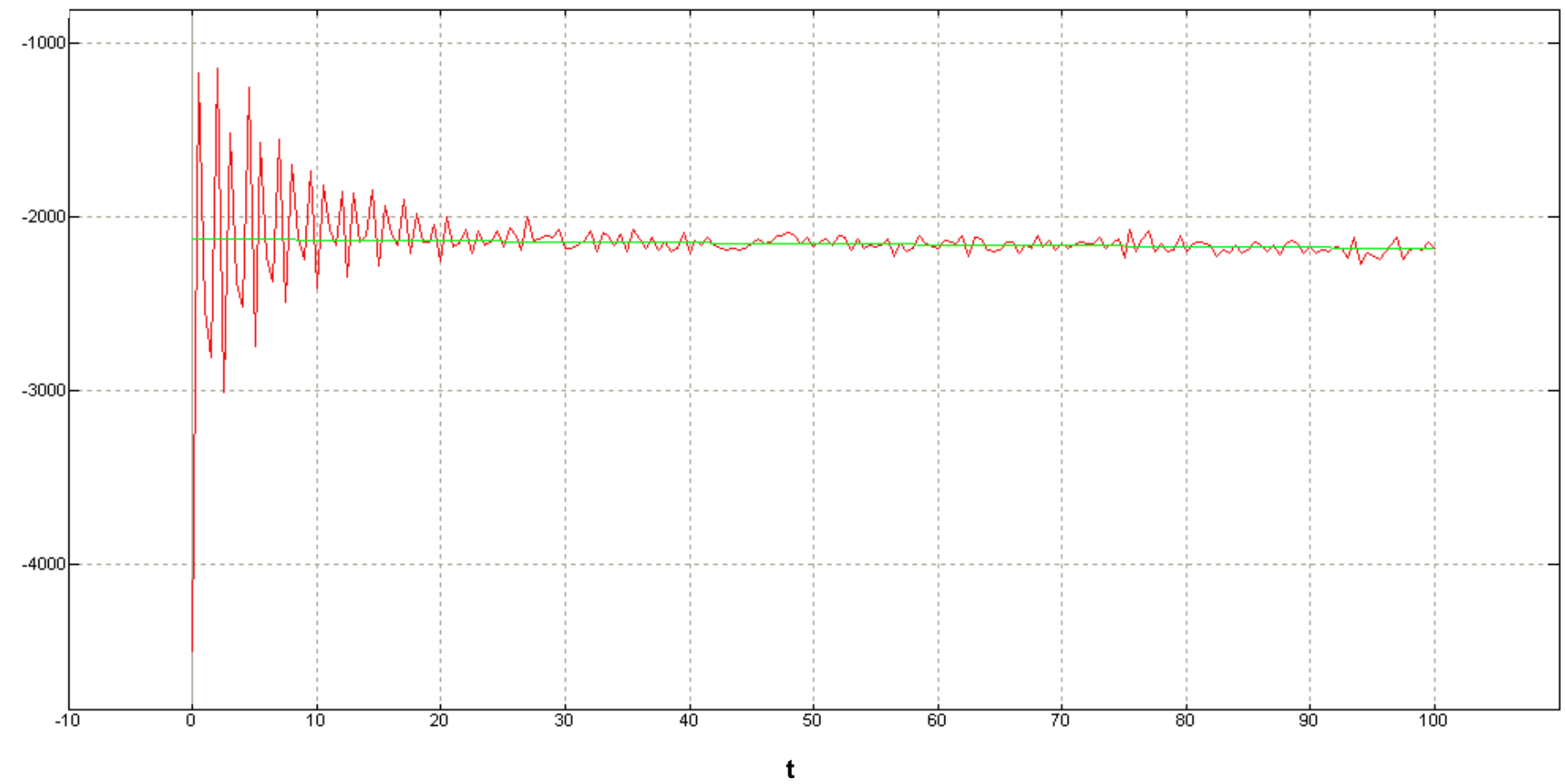

Figure 3. Correlation plot of the initialization parameter determination.

changes are also done through the root-mean-square deviation (RMSD) values. It is observed as an indication of the degree of change in backbone conformational changes in the structure of the simulation time. The triplo dynamics computation has been applied, and no significant change in free energy could be found.

\section{Analysis of ligand interactions}

\section{Molecular dynamics simulations at $300 \quad K$ temperature}

Interactions change during the simulation at a temperature of $300 \mathrm{~K}$ is shown in Table 1 of online supplementary material (spl mtr). Observations of KKR ligands at 1000 ps do not show hydrogen bonding with the active side. Similarly, ligand RKK, observed at 1500, 2000, $3000,3500,4000$ and 4500 ps indicates the absence of hydrogen bonds formed on the active side residues. Therefore, the two ligands are not feasible to use as an inhibitor of the protease complex NS2B-NS3 dengue virus. RGR and KRK ligand which is a ligand-based cyclic disulfide bridge, at the end of the simulation is observed to interact with Asp75 which is the active side residue. Ligand RGR did not record any interaction of hydrogen bonds at the time of 2500,3000 and 4500 ps. So, the inhibition ability decreased in that time interval. Nonetheless, it is with KRK ligand that the ligand recorded a release of hydrogen bonding on the active side at an interval of $1500,3500,4000$ and 4500 ps. Thus, ligands RGR and RRA did not have the consistency of affinity towards the active side residues. So, it may not be filed as an inhibitor for drug development. Observation of the ligand and the RKR and RRA that are also ligand-based cyclic disulfide bridges were found to have the best affinity ligand than the other five. Based on the molecular dynamics simulations at temperatures of $300 \mathrm{~K}$, the generated data of ligand RRA and RKR had a good affinity to the enzyme's active site. 3D visualization of the RRA and RKR ligand at the end of the simulation can be seen in Figure 4. From these images, it can be seen clearly that the RRA ligand can interact with the active side residues of Asp75 through three hydrogen bonds with each bond length $1.71,1.50$, and $1.86 \AA$. This case also occurred in RKR ligand that has two hydrogen bonds with Asp75 residue, having bond length of 1.65 and $1.62 \AA$, respectively. Table 1 (spl mtr) also demonstrates the interaction between the ligand and several other amino acid residues, which include Asp129. Amino acid residue Asp129 is recorded as having hydrogen bonding interactions with side chains of the ligand's amino acids arginine. Asp129 is an alkaline amino acid, so it fits with alkaline arginine and lysine as well. This is consistent with that proposed by $\mathrm{Li}$ et al. (2005) that amino acid Asp129 plays an important role in stabilizing amino acids such as arginine and lysine in the ligand. In fact, the ligand RRA and RKR recorded the greatest 
a)

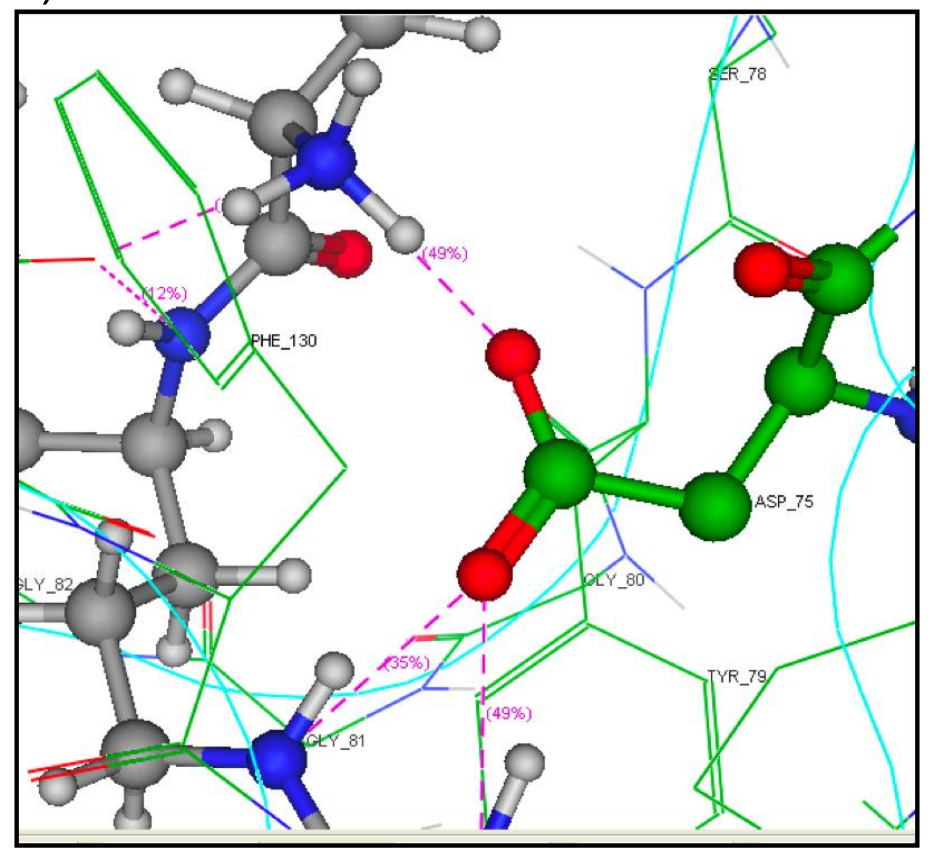

b)

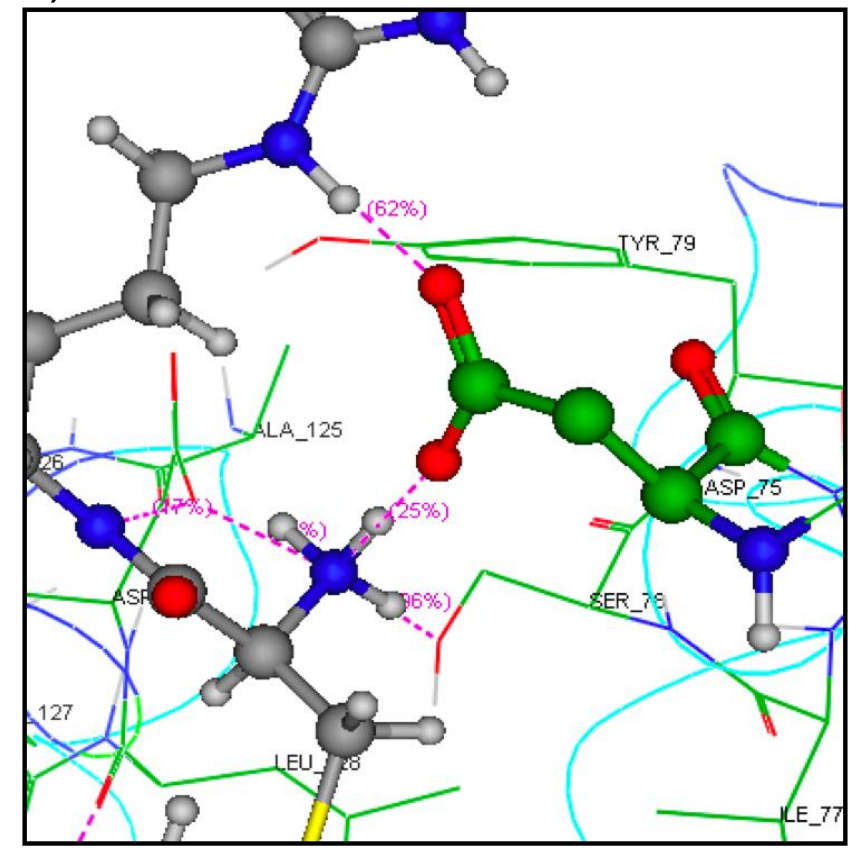

Figure 4. Visualization of 3D simulation of hydrogen bonds at a temperature of $300 \mathrm{~K}$ : (a) Ligand RRA; (b) Ligand RKR.

number of contacts with the Asp129 residue of the protease. This indicates that the ligand is suitable and stable on the active side of the NS3 protease of dengue virus.

Conformation can be seen from its frame at each time interval. RMSD results will be plotted in a graph between the RMSD and the simulation time. Conformation of the seven ligands at the time of the simulation is shown in Table 2 (spl $\mathrm{mtr}$ ). In a study presented by Zuo et al. (2009), the residues of the active NS3 protease domain is divided into two parts. Domain I consists of amino acids Asp75 and His51, while the second domain consists of amino acids Ser135. The presence of NS2B as a cofactor of the NS2 protease will surround the area, especially on the active side. In Figure 4, the area circled in red shows that the active or binding site is shaped like a slit. RMSD values in Table 3 indicate that there is a position shift of the conformation at the end of the simulation with the initial position. Free enzyme conformations have the largest shift value when compared with the complex formed between the ligand and the enzyme. Therefore, we can conclude that the presence of inhibitors in complex NS2B-NS3 protease can enhance the stability of conformations.

\section{Molecular dynamics simulation on the temperature of $312 \mathrm{~K}$}

Molecular dynamics simulations were performed at a temperature of $312 \mathrm{~K}$ to see the affinity of the ligand to the active enzyme under conditions of fever. Changes in the interaction between the ligand and the enzyme were observed to obtain the best ligand affinity. Table 3 (spl $\mathrm{mtr}$ ) shows the interaction of the ligand in the form of residue contacts and hydrogen bonds for molecular dynamics simulations at a temperature of $312 \mathrm{~K}$. The treatment was performed for the system to represent the temperature at the time of fever. In Table 3 (spl mtr), the data obtained from the different interactions are generated through the simulation at a temperature of $300 \mathrm{~K}$. Overall, all ligands (except the standard ligand) can interact with the enzyme active site through contact residues and hydrogen bonds at the end of the simu0lation. This simulation also produces a conformational change that can be observed in Table 4 (spl mtr).

Significant changes occur when the complex is treated by raising the temperature of the system from $300 \mathrm{~K}$ to $312 \mathrm{~K}$. Thus, the observation of the complex interactions between the ligand is able to provide information to study the affinity of an inhibitor.In Table 4 (spl mtr), it can be observed from the cleft position that the enzyme binding site area was formed during the simulation in progress. In Table 4, it is also shown that the ligand RRA and RKR at the end of the simulation bound to the binding site area. However, this position is covered by the conformation of the formed enzyme.

Thus, the catalytic function of the en-zymes is lost. Observations on the ligand KKR, RKK, RGR and KRK also showed that the ligand binding area remained on the site of NS2B-NS3 protease complex. In general, the conformation of the enzyme complex formed between the 
Table 3. RMSD in the simulation with the temperature of $300 \mathrm{~K}$.

\begin{tabular}{lc}
\hline Ligand & RMSD $(\AA ̊ \AA)$ \\
\hline Free Enzyme & 18.816 \\
Cyclic (-Cys-RKR-Cys-) & 14.357 \\
Cyclic (-Cys-RRA-Cys-) & 12.294 \\
Cyclic (-Cys-KRK-Cys-) & 11.227 \\
Cyclic (-Cys-RGR-Cys-) & 11.196 \\
Cyclic (-Pro-RKK-Pro-) & 12.516 \\
Cyclic (-Pro-KKR-Pro-) & 11.743 \\
Bz-Nle-Lys-Arg-Arg-H & 13.358 \\
\hline
\end{tabular}

inhibitor always changes during the simulation. This change is also indicated by the RMSD values in Table 4 .

From Table 4, significant differences can be seen when compared with the RMSD values on the simulation with the temperature of $300 \mathrm{~K}$. On the free-enzyme state, a decline in the value of RMSD from $18.816 \AA$ to $14.243 \AA$ was noted. However, this case is different from what happened in the other six ligands.

The six ligands are likely to experience changes that increase the value of RMSD. The ligand conformation of RKR and RRA is fitted into the slit on the enzyme, resulting in an enzyme binding site which is also bent into the catalytic site. Visualization of the active conformation of the ligand, and the RRA and RKR molecular dynamics simulation results at a temperature of $312 \mathrm{~K}$ can also be observed in Figure 5. In Figure 5, it is observed that the RRA ligands can form three hydrogen bonds with residues Asp75 to the active side. The bond lengths are $1.54,1.70$, and $1.90 \AA$, respectively. While the visualization of the RKR ligand was observed, it has four hydrogen bonds each with the lengths of $1.53,1.60,1.81$ and $1.93 \AA$, respectively. It can be concluded that the RKR ligand interaction with the active site of NS3 protease in molecular dynamics simulations with a temperature of $312 \mathrm{~K}$ was the best. This is because the RKR ligand is the most capable of forming hydrogen bonds when compared with most of the other ligands.

\section{Combination of molecular docking and molecular dynamics simulation}

In this study, both docking and dynamics methods are combined to see the interaction between the ligand as an inhibitor to the enzyme complex. This combination produces a data comparison of the interaction between the ligand during docking and dynamics simulation in Table 4.

From Table 4, it can be observed that the standard ligand docking process has a hydrogen bond with the active side residues Asp75, His51 and Ser135. Observations at a temperature of $300 \mathrm{~K}$ and $312 \mathrm{~K}$ show that at
Table 4. The RMSD values based on the simulation with a temperature of $312 \mathrm{~K}$.

\begin{tabular}{ll}
\hline Ligand & RMSD (̊̊) \\
\hline Free Enzim & 14.243 \\
Siklik (-Cys-RKR-Cys-) & 17.446 \\
Siklik (-Cys-RRA-Cys-) & 14.370 \\
Siklik (-Cys-KRK-Cys-) & 17.624 \\
Siklik (-Cys-RGR-Cys-) & 12.810 \\
Siklik (-Pro-RKK-Pro-) & 15.446 \\
Siklik (-Pro-KKR-Pro-) & 15.375 \\
Bz-Nle-Lys-Arg-Arg-H & 13.354 \\
\hline
\end{tabular}

the end of the simulation, the standard ligand has lost its inhibition ability. Simulation at a temperature of $300 \mathrm{~K}$ for ligand RKR and RRA showed that the side chains of arginine can interact with Asp75 residue. Similarly, the hydrogen bonding interaction is seen at a temperature of $312 \mathrm{~K}$, such that the arginine side chain can influence the formation of hydrogen bonds.

In a study conducted by Wichapong et al. (2009), arginine and lysine residues are part of residue P2 which forms the inhibitor. This P2 residue would interact with the S2 pocket of the enzyme, including the active side residues of Asp75. For the $\mathrm{P} 1$ residue of the inhibitor, it is also proposed that the arginine plays an important role in the interaction with the S2 pocket, which is the active Ser135 residue.

Lysine residues can also be selected as the P3 of the inhibitor to bind to the S3 pocket residues. The interaction between the inhibitor in the pocket P4 and S4 in the NS3 protease is dominated by hydrophobic interactions such as Val154 and Val155 (Li et al., 2005). Further visualization can be seen in Figure 6. RKK and KKR ligands that are cyclic proline-proline-based ligands also show that the hydrogen bond formed by residues Asp75 is a form of interaction of the side chains of arginine or lysine. Observation of the ligand and the RGR at both temperatures showed that RRA form similar interactions with other ligands (Tambunan et al., 2010).

The depiction of the seven ligands resulting from each process can be observed in Table 5 (spl mtr). The results of molecular docking processes compared with the molecular dynamics simulation at 300 and $312 \mathrm{~K}$ produced a difference in conformation of the enzyme complex.

It showed that the enzyme binding site area did not change as long as the simulation did not change. So it formed a cleft as the substrate binding site. However, the movement did not make the drug to be inactive towards the active side of the target (Tambunan et al., 2010). It was observed that at the end of the simulation, all the ligands except the standard one were able to interact with the active side residue of NS3 protease via hydrogen bonds. 
a)

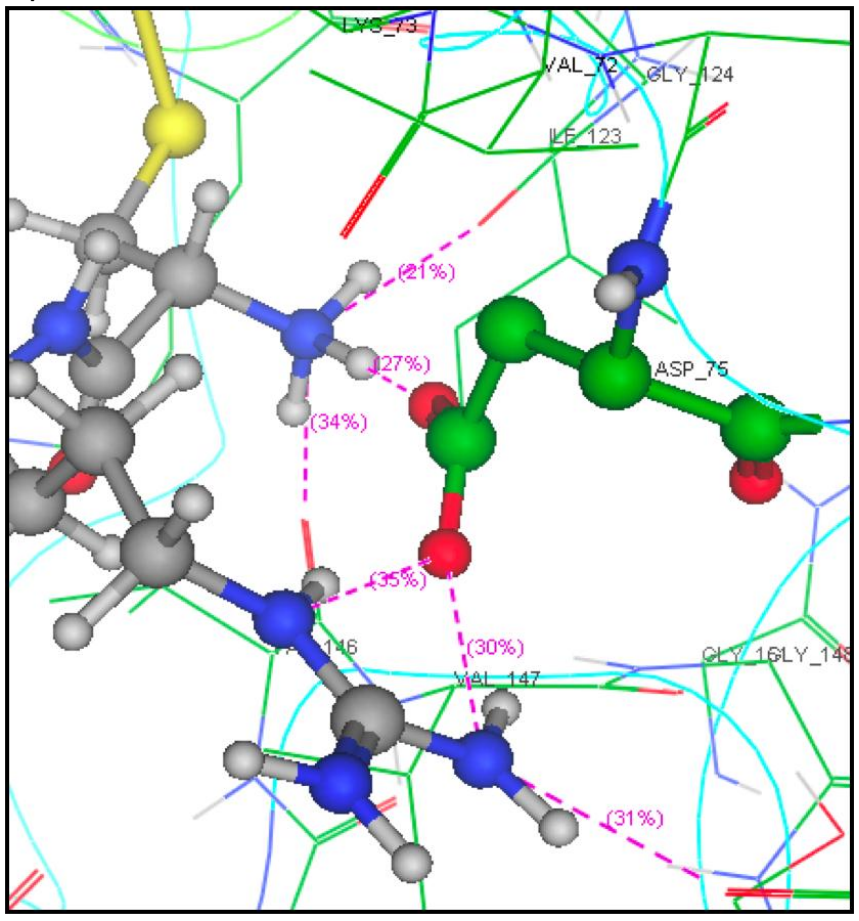

b)

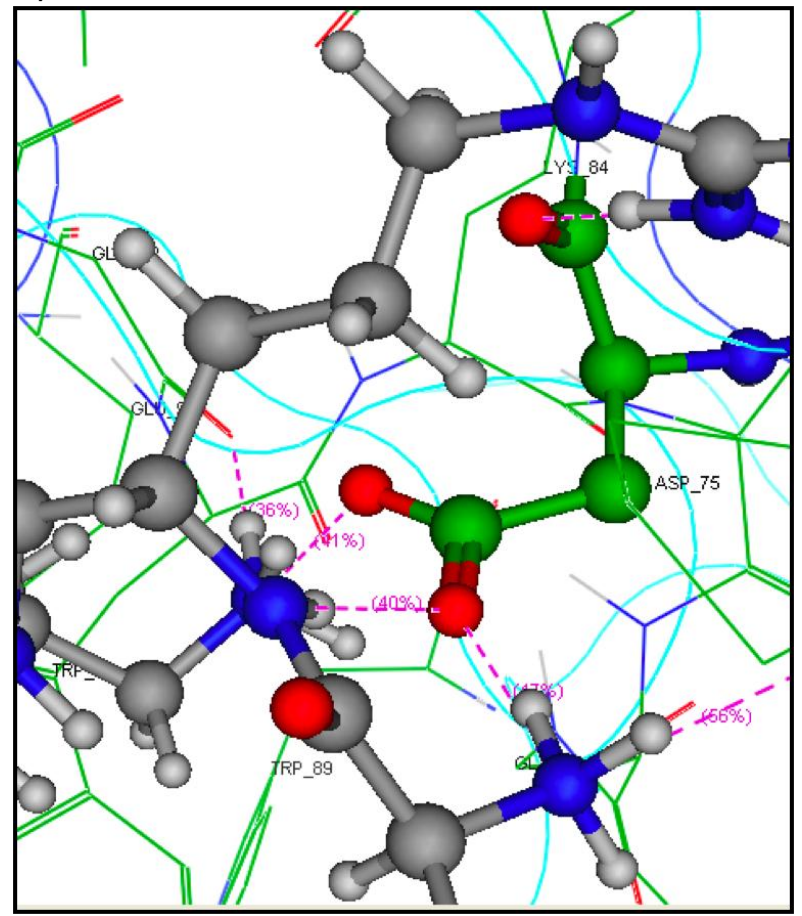

Figure 5. Visualization of 3D simulation of hydrogen bonds at a temperature of $312 \mathrm{~K}$. (A) Ligand RRA; (b) Ligand RKR.

\section{Conclusion}

Based on studies using molecular dynamics simulation method, results were obtained that the interaction of the amino acid residues of NS2B-NS3 protease ligand of dengue virus type 2 is affected by the behavior of the dynamic movement of the enzyme-ligand complex. This dynamic movement occurs due to the influence of explicit solvent in the system.

The influence of this dynamic movement, among others, are the residue contact interactions and hydrogen bonding between the ligand and the enzyme amino acid residues. This dynamic movement also influences the shape of conformations generated during the simulation. Molecular docking results showed seven residues of the ligand that can interact with the active enzyme. This process produces a ligand RKR in the top position with a minimum value of $\Delta \mathrm{G}_{\text {binding }}$ and the largest $\mathrm{PK}_{\mathrm{i}}$. Having performed molecular dynamics simulations, all ligands except the standard have an active side residue of NS3 protease.

However, the relatively stable ligands that bind to the active side are the RRA and RKR. At the end of the simulation temperature of $300 \mathrm{~K}$, ligands RRA and RKR have hydrogen bonding contacts with Asp75. Then at the end of the simulation temperature of $312 \mathrm{~K}$, ligands RKR and RRA also have hydrogen bonds with Asp75. Therefore, the determination of the best ligand was done by reviewing the value of $\Delta \mathrm{G}_{\text {binding }}$ molecular docking results.
Standard ligand linear conformation was not stable to attach to the binding site area when compared with other cyclic ligands. This is evident from the shift experienced by the ligand conformation standard of the binding site area during the simulation. RKR ligand conformation was observed to have the most suitable form of the binding site area. Conformations that are formed in the enzymeinhibitor complex also changes during the simulation. RKR has the conformation of the ligand due to the suitability of the alkaline side chain structure that is appropriate for a negatively charged cleft (oxyanion hole). Based on the analysis of ligand interactions and conformation, the ligand affinity RKR is the best compared to other ligands.

Thus, the combination of molecular docking methods and molecular dynamics simulation produces the best RKR ligand that has potential to be developed as a drug. Further study needs to be conducted on the absorption, distribution, metabolism, excretion, and toxi-city (ADMET) of ligands proposed as a drug as well as considering the possibility of a mutation in the enzyme due to binding of the ligand.

\section{ACKNOWLEDGEMENT}

The authors are grateful to Hibah Riset Utama DRPM UI (Grant Number: DRPM/RII/224/RU-UI/2013) for supporting this research. This research was supervised by 
Table 5. Interaction of the ligand amino acid residues with molecular docking and molecular dynamics simulation at a temperature of $300 \mathrm{~K}$ and $312 \mathrm{~K}$.

\begin{tabular}{|c|c|c|c|}
\hline Ligand & Docking result & $300 \mathrm{~K}$ & $312 \mathrm{~K}$ \\
\hline RKR & $\begin{array}{l}\text { Trp50, Val52, Asp75, Pro132, } \\
\text { Gly133, His51, Lys73, Asn152, } \\
\text { Gly153, Val162, Val154, Tyr161, } \\
\text { Leu128 }\end{array}$ & $\begin{array}{l}\text { Tyr79, Asp75, Asp129, Ser78, } \\
\text { Leu128, Phe130, Ala38, Ser131, } \\
\text { Val40, Gly43 }\end{array}$ & $\begin{array}{l}\text { Leu115, Phe116, Thr118, Thr48, } \\
\text { Asp71, Asp75, Pro67, Val72, } \\
\text { Lys74, Gly82, Trp83 }\end{array}$ \\
\hline RRA & $\begin{array}{l}\text { Pro132, His51, Gly153, Asn152, } \\
\text { Lys73, Asp75, Val72, Trp50, Arg54 }\end{array}$ & $\begin{array}{l}\text { Trp83, Gly82, Gly81, Gly151, } \\
\text { Asp129, Tyr79, Asp75, Tyr150, } \\
\text { Ser78, Ile139, Ser127, Leu128 }\end{array}$ & $\begin{array}{l}\text { Thr45, Gly44, Phe46, Met49, } \\
\text { His47, Val140, Asp141, Gly103, } \\
\text { Val146, Lys142, Asp75 }\end{array}$ \\
\hline KRK & $\begin{array}{l}\text { Arg54, Asp75, Gly153, Asn152, } \\
\text { Leu128, Ser135, Tyr150, Gly151, } \\
\text { Pro132, His51, Gly133, Val52, Ile36 }\end{array}$ & $\begin{array}{l}\text { Glu66, Asp71, Asp75, Tro50, } \\
\text { Lys74, Arg54, Val52, Val72, Gly55 }\end{array}$ & $\begin{array}{l}\text { Trp61, Ala65, Glu48, Ser71, } \\
\text { Gly69, Ala49, Ser70, Leu74, } \\
\text { Pro67, Trp69, Ser68, Glu66, } \\
\text { Arg64, Asp75, Leu76, Asp71, } \\
\text { Trp50, Arg54, Glu66 }\end{array}$ \\
\hline RGR & $\begin{array}{l}\text { GIn35, Glu92, lle36, Pro132, Val52, } \\
\text { His51, Arg54, Asp75, Asn152, } \\
\text { Gly153 }\end{array}$ & Asp75, Trp50, Glu66, Leu76, Ile77 & $\begin{array}{l}\text { Ser158, Val154, His51, Trp50, } \\
\text { Tyr79, Asp75, Leu76, Arg54 }\end{array}$ \\
\hline RKK & $\begin{array}{l}\text { Val52, His51, Leu128, Ser163, } \\
\text { Gly151, Asn152, Asp75, Gly153, } \\
\text { Lys74, Lys73, Val72 }\end{array}$ & $\begin{array}{l}\text { His47, Phe46, Gly39, Ala38, } \\
\text { Leu149, Asn152, Tyr150, Ser135, } \\
\text { Asp129 }\end{array}$ & $\begin{array}{l}\text { Asp75, Gly153, Asn152, GIn93, } \\
\text { Gly96, Tyr161, Gly37, Ile36, } \\
\text { Ser158, Val155, Arg157, Ser78 }\end{array}$ \\
\hline $\mathrm{KKR}$ & $\begin{array}{l}\text { Val72, Asp75, Asn152, Trp50, } \\
\text { Gly153, His51, Ile36, Pro132, } \\
\text { Gly133 }\end{array}$ & $\begin{array}{l}\text { Asn152, Asn119, Thr122, Tyr79, } \\
\text { Asp75, Ser78 }\end{array}$ & $\begin{array}{l}\text { Asp75, Tyr79, Gly148, Asp129, } \\
\text { Ser131, Met49, Ser131, Pro132, } \\
\text { Ser135, His47, Val147, Tyr79 }\end{array}$ \\
\hline Standar & $\begin{array}{l}\text { Arg54, Asp75, Val52, Ile36, His51, } \\
\text { Gly133, GIn27, Ser135, Pro132, } \\
\text { Gly153, Asn152 }\end{array}$ & $\begin{array}{l}\text { Lys104, Gly103, Ser131, Gln110, } \\
\text { Ser127, Ser70, Arg107, Ser68, } \\
\text { Pro106, Phe130 }\end{array}$ & $\begin{array}{l}\text { Glu90, Lys28, Ser85, Ile86, } \\
\text { Glu101, Glu89, GIn27 }\end{array}$ \\
\hline
\end{tabular}

Bold, Amino acid residues that facilitate hydrogen bonding with the ligand; Bold, active site residues that facilitate hydrogen bonding with the ligand.

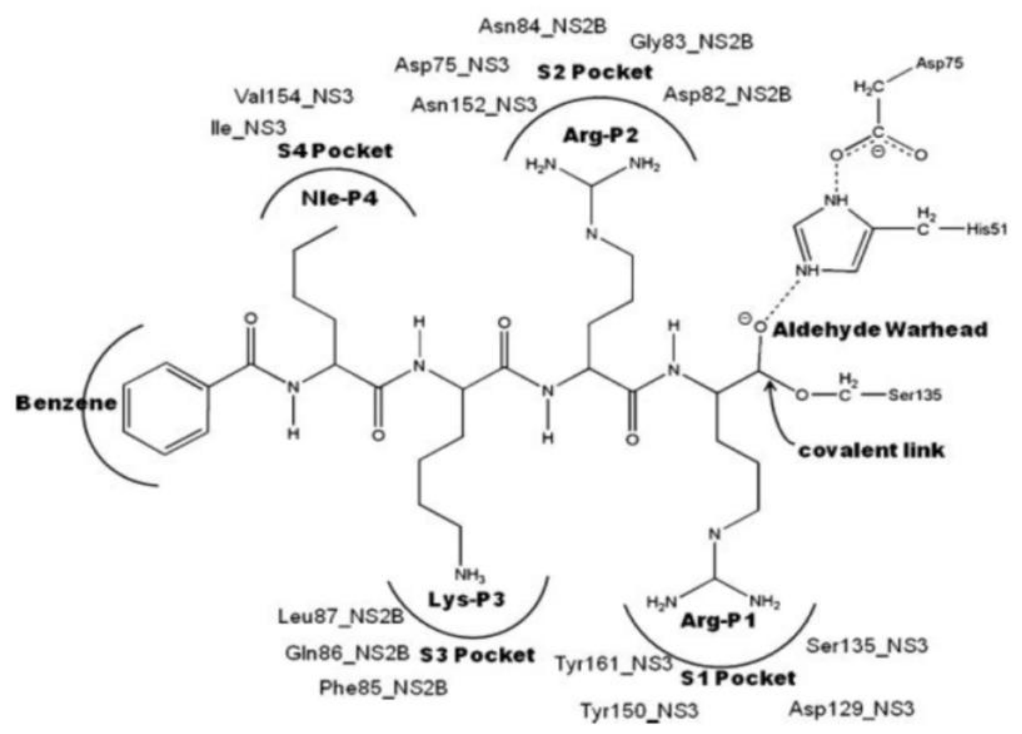

Figure 6. Visualization of interaction between $\mathrm{P}$ (ligand) and $\mathrm{S}$ (enzyme) (Wichapong et al., 2009). 
Usman Sumo Friend Tambunan while the technical details were processed by Irwan Kurniawan. However, Arli Aditya Parikesit prepared the english manuscript and verified the data.

\section{REFERENCES}

Alonso H, Bliznyuk AA, Gready JE. (2006). Combining Docking and Molecular Dynamic Simulations in Drug Design. Med. Res. Rev. Wiley InterScience. 26(5):531-68. doi: 10.1002/med.20067.

Bollati M. (2010). Structure and functionality in flavivirus NS-proteins: Perspectives for drug design. Antivir. Res. 87:125-148. Science Direct. Elsevier.

Erbel PI, et al. (2006). Structural basis for the activation of flaviviral NS3 proteases from dengue and West Nile virus. Nat. Struct. Mol. Biol. 13(4):372-3. doi:10.1038/nsmb1073.

Jois SD, Tambunan US, Chakrabarti S, Siahaan TJ. (1996). Solution structure of a cyclic RGD peptide that inhibits platelet aggregation. J. Biomol. Struct. Dyn. 14(1):1-11.

Katzenmeier G. (2004). Inhibition of the NS2B-NS3 Protease - Towards a Causative Therapy for Dengue Virus Diseases. Dengue Bull. Vol 28

Kirsten G. (2008). Concept of Pharmacophores and their Application in Computer Aided Drug Design. ICS UNIDO Workshop.Trieste, Italy

Leach AR. (2001). Molecular Modelling: Principles and Application. Second Edition. Prentice Hall. New Jersey.

Lescar J, Luo D, et al. (2008). Towards the design of antiviral inhibitors against flaviviruses: The case for the multifunctional NS3 protein from dengue virus as a target. Antivir. Res. 80:94-101.

Leung D, Schroder K, White H, Fang NX, Stoermer MJ, Abbenante G, Martin JL, Young PR, Fairlie DP. (2001). Activity of recombinant Dengue 2 virus NS3 protease in the presence of a truncated NS2B co-factor, small peptide substrates, and inhibitors. J. Biol. Chem. 276(49):45762-45771

Li J, Lim SP, Beer D, Patel V, et al., (2005). Functional Profiling of Recombinant NS3 Proteases from All Four Serotypes of Dengue Virus Using Tetrapeptide and Octapeptide Substrate Libraries. J. Biol. Chem. 280(31):28766-74. doi: 10.1074/jbc.M500588200

Noble C, et al. (2009). Strategy for development of dengue virus Inhibitors. Antivir. Res. 85(3):450-62. Elsevier.

Nurbaiti S, Nagao H, Saito H, Hertiadi R, Martoprawiro MA et al. (2010). Domain motions of Klenow-like DNA polymerase I ITB-1 in the absence of substrate. Int. J. Integrative Biol. 9:04-110.

Nylander E. (2007). DockControl: A New Integrated Software for Design of Experiments and Molecular Docking: Application to HIV-Protease Inhibitors. Msc Thesis. Department of Computer Science. Umea University. Sweden.
Online First Supplementary Material : http://www.bioinf.unileipzig.de/ arli/supplementary_material_ir.docx

Qi R-F, Zhang L, Chi C-W. (2008). Biological characteristics of dengue virus and potential targets for drug design. Acta Biochim Biophys. Sin., Vol: 40, Pp. 91-101.

Sampath et al., (2009). Molecular targets for flavivirus drug discovery. Antivir. Res. 81(1):6-15. Doi. 10.1016/j.antiviral.2008.05.004 Elsevier.

Sharma et al. (2009). High temperature unfolding of Bacillus anthracis amidase- 03 by molecular dynamics simulations. Bioinformation $3(10): 430-434$

Singh S, et al. (2006). Molecular drug targets and structure based drug design: A holistic Approach. Bioinformation. 1(8):314-320.

Tambunan US, Alamudi S. (2010). Designing cyclic peptide inhibitor of dengue virus NS3-NS2B protease by using molecular docking approach. Bioinformation 5(6):250-254.

Tambunan US, Parikesit AA, Hendra, Taufik RI, Amelia F, Syamsudin. (2009). In Silico Analysis of E DENV-2 and E DENV-3 Protein as the Backbone of Dengue Virus Tetravalent Vaccine by Using Homology Modeling Method. OnLine J. Biol. Sci. 9(1):6-16

Tomlinson SM, Malmstrom RD, Watowich SJ. (2009). New Approaches to Structure-Based Discovery of Dengue Protease Inhibitors. Infect. Disord. Drug Targets.9(3):327-43.

Vilar S, Cozza G, Moro S. (2008). Medicinal chemistry and the molecular operating environment (MOE): application of QSAR and molecular docking to drug discovery. Curr. Top. Med. Chem. 8(18):1555-1572.

WHO (2010). Dengue and dengue haemorrhagic fever. World Health Organization.

Wichapong K, Somsak P, et al. (2009). Homology modeling and molecular dynamics simulations of dengue virus NS2B/NS3 protease: insight into molecular interaction. J. Mol. Recognit. 23(3):283-300. doi:10.1002/jmr.977

Yin Z, Patel SJ, Wang WL, et al. (2005). Peptide inhibitors of dengue virus NS3 protease. Part 2:SAR study of tetrapeptide aldehyde inhibitors. Bioorg. Med. Chem. Lett. 16(1):40-3

Yusof R, Clum S, Wetzel M, Murthy HM, Padmanabhan R. (2000). Purified NS2B/NS3 serine protease of Dengue virus type 2 exhibits cofactor NS2B dependence for cleavage for substrates with dibasic amino acids in vitro. J. Biol. Chem. 275(14):9963-9969.

Zuo et al. (2009).Mechanism of NS2B-Mediated Activation of NS3pro in Dengue Virus: Molecular Dynamics Simulations and Bioassays. J. Virol., P. 1060-1070 Vol. 83, No. 20022-538x/09/\$08.00 0 Doi:10.1128/Jvi.01325-08. 